\title{
Analysis of C9orf72 repeat expansions in a large international cohort of dementia with Lewy bodies
}

\section{Kun-Rodrigues, Celia}

2017-01

Kun-Rodrigues , C , Ross , O A , Orme , T, Shepherd, C, Parkkinen , L, Darwent , L , Hernandez, D , Ansorge , O, Clark , L N, Honig , L S, Marder , K, Lemstra, A, Scheltens , P , van der Flier , W , Louwersheimer , E , Holstege , H, Rogaeva , E , St George-Hyslop , P , Londos , E , Zetterberg , H, Barber , I, Braae , A, Brown , K, Morgan, K, Maetzler , W , Berg , D , Troakes , C , Al-Sarraj , S, Lashley , T, Holton, J, Compta , Y, Van Deerlin , V , Trojanowski , J Q , Serrano, G E , Beach , T G , Clarimon , J , Lleo , A ,

Morenas-Rodriguez, E, Lesage , S, Galasko, D, Masliah , E , Santana , I, Diez, M , Pastor, P , Tienari , P J , Myllykangas , L , Oinas, M , Revesz , T, Lees , A , Boeve , B F , Petersen, R C , Ferman , T J , Escott-Price , V , Graff-Radford , N , Cairns , N J , Morris , J C , Stone , D J , Pickering-Brown, S, Mann , D , Dickson, D W , Halliday , G M, Singleton , A , Guerreiro , R \& Bras , J 2017 , ' Analysis of C9orf72 repeat expansions in a large international cohort of dementia with Lewy bodies ', Neurobiology of Aging , vol. 49 , ARTN 214.e13 . https://doi.org/10.1016/j.neurobiolaging.2016.08.023

http://hdl.handle.net/10138/234441

https://doi.org/10.1016/j.neurobiolaging.2016.08.023

publishedVersion

Downloaded from Helda, University of Helsinki institutional repository.

This is an electronic reprint of the original article.

This reprint may differ from the original in pagination and typographic detail.

Please cite the original version. 
Negative results

\section{Analysis of C9orf72 repeat expansions in a large international cohort of dementia with Lewy bodies}

Celia Kun-Rodrigues ${ }^{\mathrm{a}}$, Owen A. Ross ${ }^{\mathrm{b}}$, Tatiana Orme ${ }^{\mathrm{a}}$, Claire Shepherd ${ }^{\mathrm{c}, \mathrm{d}}$, Laura Parkkinen ${ }^{\mathrm{e}}$, Lee Darwent ${ }^{\mathrm{a}}$, Dena Hernandez ${ }^{\mathrm{f}}$, Olaf Ansorge ${ }^{\mathrm{e}}$, Lorraine N. Clark ${ }^{\mathrm{g}, \mathrm{h}}$, Lawrence S. Honig ${ }^{g, h}$, Karen Marder ${ }^{\text {g,h }}$, Afina Lemstra ${ }^{\mathrm{i}}$, Philippe Scheltens ${ }^{\mathrm{i}}$, Wiesje van der Flier ${ }^{i}$, Eva Louwersheimer ${ }^{i}$, Henne Holstege ${ }^{i}$, Ekaterina Rogaeva ${ }^{j}$, Peter St. George-Hyslop ${ }^{\mathrm{j}}$, Elisabet Londos ${ }^{\mathrm{k}}$, Henrik Zetterberg ${ }^{1, \mathrm{~m}}$, Imelda Barber ${ }^{\mathrm{n}}$, Anne Braae ${ }^{\mathrm{n}}$, Kristelle Brown ${ }^{\mathrm{n}}$, Kevin Morgan ${ }^{\mathrm{n}}$, Walter Maetzler ${ }^{\mathrm{o}, \mathrm{p}}$, Daniela Berg ${ }^{\mathrm{o}, \mathrm{p}}$, Claire Troakes q, Safa Al-Sarraj q, Tammaryn Lashley ${ }^{\mathrm{r}}$, Janice Holton ${ }^{\mathrm{r}}$, Yaroslau Compta ${ }^{\mathrm{s}}$, Vivianna Van Deerlin ${ }^{\mathrm{t}}$, John Q. Trojanowski ${ }^{\mathrm{t}}$, Geidy E. Serrano ${ }^{\mathrm{u}}$, Thomas G. Beach ${ }^{\mathrm{u}}$, Jordi Clarimon ${ }^{\mathrm{v}}$, Alberto Lleó ${ }^{\mathrm{v}}$, Estrella Morenas-Rodríguez ${ }^{\mathrm{v}}$, Suzanne Lesage ${ }^{\mathrm{w}, \mathrm{x}}$, Douglas Galasko $^{\mathrm{y}, \mathrm{z}}$, Eliezer Masliah ${ }^{\mathrm{y}, \mathrm{z}}$, Isabel Santana ${ }^{\mathrm{aa}}$, Monica Diez ${ }^{\mathrm{bb}, \mathrm{cc}}$, Pau Pastor ${ }^{\mathrm{bb},{ }^{c c}}$, Pentti J. Tienari ${ }^{\mathrm{dd}}$, Liisa Myllykangas ${ }^{\mathrm{ee}}$, Minna Oinas ${ }^{\mathrm{ff}}$, Tamas Revesz ${ }^{\mathrm{r}}$, Andrew Lees ${ }^{r}$, Brad F. Boeve ${ }^{g g}$, Ronald C. Petersen ${ }^{g g}$, Tanis J. Ferman ${ }^{\text {hh, }}$,ii, Valentina Escott-Price ${ }^{\mathrm{jj}}$, Neill Graff-Radford ${ }^{\mathrm{kk}}$, Nigel J. Cairns ${ }^{11}$, John C. Morris ${ }^{11}$, David J. Stone $^{\mathrm{mm}}$, Stuart Pickering-Brown ${ }^{\mathrm{nn}}$, David Mann ${ }^{\mathrm{nn}}$, Dennis W. Dickson ${ }^{\mathrm{b}}$, Glenda M. Halliday ${ }^{\mathrm{c}, \mathrm{d}}$, Andrew Singleton ${ }^{\mathrm{f}}$, Rita Guerreiro ${ }^{\mathrm{oo}}$,pp, Jose Bras ${ }^{\mathrm{oo}, \mathrm{pp} \text {,* }}$

\footnotetext{
a Department of Molecular Neuroscience, UCL Institute of Neurology, London, UK

${ }^{\mathrm{b}}$ Department of Neuroscience, Mayo Clinic, Jacksonville, FL, USA

${ }^{\mathrm{c}}$ Neuroscience Research Australia, Sydney, Australia

d School of Medical Sciences, Faculty of Medicine, University of New South Wales, Sydney, Australia

e Nuffield Department of Clinical Neurosciences, Oxford Parkinson's Disease Centre, University of Oxford, Oxford, UK

${ }^{\mathrm{f}}$ Laboratory of Neurogenetics, National Institutes on Aging, NIH, Bethesda, MD, USA

${ }^{g}$ Taub Institute for Alzheimer Disease and the Aging Brain, Columbia University, New York, NY, USA

${ }^{\mathrm{h}}$ Department of Pathology and Cell Biology, Columbia University, New York, NY, USA

${ }^{i}$ Department of Neurology and Alzheimer Center, Neuroscience Campus Amsterdam, VU University Medical Center, Amsterdam, the Netherlands

${ }^{\mathrm{j}}$ Department of Medicine, Tanz Centre for Research in Neurodegenerative Diseases, University of Toronto, Toronto, Ontario, Canada

${ }^{\mathrm{k}}$ Clinical Memory Research Unit, Institution of Clinical Sciences Malmö, Lund University, Lund, Sweden

${ }^{1}$ Department of Molecular Neuroscience, UCL Institute of Neurology, London, UK

${ }^{\mathrm{m}}$ Clinical Neurochemistry Laboratory, Institute of Neuroscience and Physiology, Sahlgrenska Academy at the University of Gothenburg, Mölndal, Sweden

${ }^{\mathrm{n}}$ Translation Cell Sciences-Human Genetics, School of Life Sciences, Queens Medical Centre, University of Nottingham, Nottingham, UK

${ }^{\circ}$ Hertie Institute for Clinical Brain Research, Department of Neurodegeneration, Center of Neurology, University of Tuebingen, Tuebingen, Germany

${ }^{\mathrm{p}}$ Department of Neurology, Christian-Albrechts University of Kiel, Kiel, Germany

${ }^{\mathrm{q}}$ Department of Basic and Clinical Neuroscience and Institute of Psychiatry, Psychology and Neuroscience, King's College London, London, UK

${ }^{\mathrm{r}}$ Queen Square Brain Bank, Department of Molecular Neuroscience, UCL Institute of Neurology, London, UK

${ }^{s}$ Movement Disorders Unit, Neurology Service, Clinical Neuroscience Institute (ICN), Hospital Clínic, University of Barcelona, IDIBAPS, Barcelona, Spain

${ }^{\mathrm{t}}$ Department of Pathology and Laboratory Medicine, Center for Neurodegenerative Disease Research, Perelman School of Medicine at the University of Pennsylvania, Philadelphia, PA, USA

${ }^{\mathrm{u}}$ Banner Sun Health Research Institute, Sun City, AZ, USA

${ }^{v}$ Department of Neurology, IIB Sant Pau, Hospital de la Santa Creu i Sant Pau, Universidad Autònoma de Barcelona, Barcelona, Catalonia, Spain

${ }^{\mathrm{w}}$ Sorbonne Université, Université Pierre et Marie Curie-Paris 06, Inserm, Centre National de la Reserche Scientifique, Institute du Cerveau et de la Moelle épinière, Paris, France

${ }^{\mathrm{x}}$ Assistance Publique Hôpitaux de Paris, Hôpital de la Salpêtrière, Département de Génétique et Cytogénétique, Paris, France

${ }^{y}$ Department of Neurosciences, University of California, San Diego, La Jolla, CA, USA

${ }^{z}$ Veterans Affairs San Diego Healthcare System, La Jolla, CA, USA

${ }^{\text {aa }}$ Centro Hospitalar e Universitário de Coimbra, Coimbra, Portugal
}

\footnotetext{
* Corresponding author at: Department of Molecular Neuroscience, 1 Wakefield Street (1st Floor), London WC1N 1PJ, England. Tel.: +44 (0) 207679 4256; fax: +44 (0)20 78331016
} 
${ }^{\mathrm{bb}}$ Memory Unit, Department of Neurology, University Hospital Mútua de Terrassa, and Foundation Mútua de Terrassa, Barcelona, Spain

${ }^{c c}$ Centro de Investigación Biomédica en Red Enfermedades Neurodegenerativas (CIBERNED), Madrid, Spain

${ }^{\mathrm{dd}}$ Molecular Neurology, Research Programs Unit, University of Helsinki, Department of Neurology, Helsinki University Hospital, Helsinki, Finland

ee Department of Pathology, University of Helsinki, Helsinki, Finland and HUSLAB

${ }^{\mathrm{ff}}$ Department of Neuropathology and Neurosurgery, Helsinki University Hospital and University of Helsinki, Helsinki, Finland

gg Neurology Department, Mayo Clinic, Rochester, MN, USA

he Department of Psychiatry, Mayo Clinic, Jacksonville, FL, USA

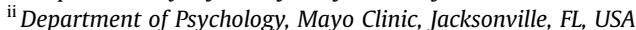

${ }_{\mathrm{ji}}$ MRC Centre for Neuropsychiatric Genetics and Genomics, School of Medicine, Cardiff University, Cardiff, UK

${ }^{\mathrm{kk}}$ Department of Neurology, Mayo Clinic, Jacksonville, FL, USA

${ }^{11}$ Knight Alzheimer's Disease Research Center, Department of Neurology, Washington University School of Medicine, St. Louis, MO, USA

$\mathrm{mm}^{\mathrm{m}}$ Genetics and Pharmacogenomics, Merck Research Laboratories, West Point, PA, USA

${ }^{\mathrm{nn}}$ Institute of Brain, Behaviour and Mental Health, Faculty of Medical and Human Sciences, University of Manchester, Manchester, UK

${ }^{\circ o}$ Department of Molecular Neuroscience, UCL Institute of Neurology, London, UK

pp Department of Medical Sciences and Institute of Biomedicine-iBiMED, University of Aveiro, Aveiro, Portugal

\section{A R T I C L E I N F O}

Article history:

Received 13 July 2016

Received in revised form 22 August 2016

Accepted 25 August 2016

Available online 2 September 2016

Keywords:

C9orf72

Dementia with Lewy bodies (DLB)

Genetic screen

\begin{abstract}
A B S T R A C T
C9orf72 repeat expansions are a common cause of amyotrophic lateral sclerosis and frontotemporal dementia. To date, no large-scale study of dementia with Lewy bodies (DLB) has been undertaken to assess the role of C9orf72 repeat expansions in the disease. Here, we investigated the prevalence of C9orf72 repeat expansions in a large cohort of DLB cases and identified no pathogenic repeat expansions in neuropathologically or clinically defined cases, showing that C9orf72 repeat expansions are not causally associated with DLB.
\end{abstract}

(c) 2016 Elsevier Inc. All rights reserved.

\section{Introduction}

Hexanucleotide repeat expansions (HREs) in a noncoding region of C9orf72 are recognized as the most common genetic cause of familial and sporadic amyotrophic lateral sclerosis, frontotemporal dementia (FTD), amyotrophic lateral sclerosis-FTD, and Huntington disease phenocopies (Beck et al., 2013; Boeve et al., 2012; Hensman Moss et al., 2014; Majounie et al., 2012c; Simon-Sanchez et al., 2012; van der Zee et al., 2013).

A normal repeat expansion shows 1 to 23 GGGGCC repeats located between exons 1a and 1b of C9orf72 (DeJesus-Hernandez et al., 2011; Renton et al., 2011). HREs identified in several neurodegenerative syndromes were found to range from 500 to 4400 repeats, but on a repeat-primed polymerase chain reaction (PCR), more than 32 repeats are often considered a pathogenic genotype (Beck et al., 2013).

C9orf72 HREs have been identified in nonmotor neurodegenerative phenotypes including Alzheimer's disease $(\mathrm{AD})$ at frequencies of 1\% (Beck et al., 2013; Harms et al., 2013; Kohli et al., 2013; Majounie et al., 2012b), although conflicting reports exist in the literature (Rollinson et al., 2012; Xi et al., 2012).

Dementia with Lewy bodies (DLB) accounts for $15 \%-25 \%$ of all dementia cases (Heidebrink, 2002). Its core features encompass cognitive impairment, fluctuating attention, parkinsonism, and recurrent visual hallucinations (Weisman and McKeith, 2007). Neuropathological diagnosis of DLB is achieved when the presence of Lewy bodies is confirmed in the cortex and the brainstem (McKeith et al., 2005). Little is known about the genetics of DLB, although molecular studies seem to point toward genetic overlaps with other neurodegenerative diseases, mainly with AD and Parkinson's disease (PD) (Bras et al., 2014; Guerreiro et al., 2016; Keogh et al., 2016; Meeus et al., 2012).

So far, the C9orf72 repeat expansion has only been genotyped in small cohorts of $\sim 100$ DLB cases or less (Geiger et al., 2016; Lesage et al., 2013; Robinson et al., 2014; Snowden et al., 2012; Yeh et al., 2013). We have recently shown in a large cohort that C9orf72 repeat expansions are not a common cause of DLB in pathologically diagnosed cases (Guerreiro et al., 2015). Here, we expand on these findings using a cohort of 1524 DLB cases.

\section{Material and methods}

Samples consisted of an international cohort of 1398 neuropathologically diagnosed DLB cases and 126 clinically diagnosed DLB cases (Supplementary Table 1). DNA was extracted from brain tissue for the neuropathologically diagnosed samples and from blood for the clinical diagnosed samples using standard procedures. We performed repeat-primed PCR according to Renton et al. (2011). Genotypes were assessed using Peak Scanner v2.0 (Applied Biosystems) with repeat expansions displaying a characteristic saw tooth pattern with a 6 base pair periodicity on analysis.

\section{Results}

Repeat mean number was $5.17( \pm 4.30$ standard deviation $)$ ranging from 1 to 58 . All except 5 samples presented less than 23 repeats in the repeat-primed PCR (Supplementary Fig. 1). Two neuropathologically diagnosed DLB samples showed 32 repeats and 1 showed 33 repeats; and 2 clinically diagnosed samples exhibited 33 and 58 repeats. These last 2 samples had been previously analyzed as part of the cohort published by Snowden et al. (2012).

\section{Discussion}

This is the first study genotyping the C9orf72 HREs in a large cohort of mainly neuropathologically diagnosed DLB samples. Within the neuropathologically defined DLB cases, we did not find any HREs above the typical threshold for pathogenicity ( $\sim 32$ repeats). This is concordant with previous studies that found no repeat expansions in 34 clinically diagnosed cases of a Taiwanese cohort or in 111 pathological DLB cases (Geiger et al., 2016; Yeh et al., 2013). Snowden et al. (2012) found 2 cases with HREs greater than 30 repeats in a study that was comprised of 102 "probable DLB" blood samples. When the same group restricted 
their analysis to include only pathologically diagnosed samples, no pathogenic repeat expansions were identified (Robinson et al., 2014).

DLB is considered to be part of a spectrum between AD and PD (Weisman and McKeith, 2007) where large C9orf72 HREs are not frequent. In $\mathrm{AD}$, it was suggested that pathogenic repeat expansions may only be associated with late onset AD (Kohli et al., 2013) or that amnesic FTD (which is easily misdiagnosed as AD) could be responsible for the low frequencies observed for $\mathrm{AD}$ (Majounie et al., 2012b). In PD, there is no evidence for a role of C9orf72 pathogenic repeat expansions (Majounie et al., 2012a; Xi et al., 2012).

Clinical symptoms in DLB can vary substantially from patient to patient and some can even overlap with less typical forms of FTD (Claassen et al., 2008), which could account for the pathogenic repeat expansions found in misdiagnosed DLB clinical cases. Furthermore, recent data suggest that the threshold for pathogenicity of HREs should be higher than the initially proposed 30 repeats (Xi et al., 2015).

In our cohort of neuropathologically diagnosed DLB samples, we found 3 cases with likely benign 32 and 33 repeats. Excluding the clinically diagnosed cases, we found no evidence of pathogenic repeat expansions. Even including the clinically diagnosed cohort, no extended repeat expansions were identified; with the longest allele exhibiting 58 repeats.

Our study shows that C9orf72 pathogenic repeat expansions are not a common cause of DLB.

\section{Disclosure statement}

Ronald C. Petersen reports consultancies with Roche, Inc, Merck, Inc, Genentech, Inc, Biogen, Inc, and Eli Lilly. Brad F. Boeve reports GE Healthcare, FORUM Pharmaceuticals, and C2N Diagnostics as research support and advisory board member of the Tau Consortium. The remaining authors report no competing interests.

\section{Acknowledgements}

This work was supported in part by the National Institutes of Neurological Disease and Stroke . Jose Bras and Rita Guerreiro are supported by fellowships from the Alzheimer's Society. Tatiana Orme is supported by a scholarship from the Lewy Body Society. For the neuropathologically confirmed samples from Australia, tissues were received from the Sydney Brain Bank, which is supported by Neuroscience Research Australia and the University of New South Wales. This study was also partially funded by the Wellcome Trust,
Medical Research Council, Canadian Institutes of Health Research, and Ontario Research Fund. The Nottingham Genetics Group is supported by ARUK and The Big Lottery Fund. The effort from Columbia University was supported by the Taub Institute, the Panasci Fund, the Parkinson's Disease Foundation, and NIH grants NS060113 (Dr Clark), P50AG008702 (P.I. Scott Small), P50NS038370 (P.I.R. Burke), and UL1TR000040 (P.I.H. Ginsberg). Dr Ross is supported by the Michael J. Fox Foundation for Parkinson's Research, NINDS R01\# NS078086. The Mayo Clinic Jacksonville is a Morris K. Udall Parkinson's Disease Research Center of Excellence (NINDS P50 \#NS072187) and is supported by The Little Family Foundation and by the Mangurian Foundation Program for Lewy Body Dementia research and the Alzheimer Disease Research Center (P50 AG016547). The work from the Mayo Clinic Rochester is supported by the National Institute on Aging (P50 AG016574 and U01 AG006786). This work has received support from The Queen Square Brain Bank at the UCL Institute of Neurology; where Dr Lashley is funded by an ARUK senior fellowship. Some of the tissue samples studied were provided by the MRC London Neurodegenerative Diseases Brain Bank and the Brains for Dementia Research project (funded by Alzheimer's Society and ARUK). This research was supported in part by both the NIHR UCLH Biomedical Research Centre and the Queen Square Dementia Biomedical Research Unit. This work was supported in part by the Intramural Research Program of the National Institute on Aging, National Institutes of Health, Department of Health and Human Services; project AG000951-12. The University of Pennsylvania case collection is funded by the Penn Alzheimer's Disease Core Center (AG10124) and the Penn Morris K. Udall Parkinson's Disease Research Center (NS053488). The authors would like to thank the Exome Aggregation Consortium and the groups that provided exome variant data for comparison. A full list of contributing groups can be found at http://exac.broadinstitute.org/about. Tissue samples from UCSD are supported by NIH grant AG05131. The authors thank the brain bank GIE NeuroCEB, the French program "Investissements d'avenir" (ANR-10-IAIHU-06). Dr Tienari and Dr Myllykangas are supported by the Helsinki University Central Hospital, the Folkhälsan Research Foundation and the Finnish Academy. This work was in part supported by the Canadian Consortium on Neurodegeneration in Aging (ER).

\section{Appendix A. Supplementary data}

Supplementary data related to this article can be found at http:// dx.doi.org/10.1016/j.neurobiolaging.2016.08.023. 